\title{
A Structurally Robust Chiral Borate Ion: Molecular Design, Synthesis, and Asymmetric Catalysis
}

\author{
Daisuke Uraguchi, ${ }^{1}$ Fumito Ueoka, ${ }^{1}$ Naoya Tanaka, ${ }^{1}$ Tomohito Kizu, ${ }^{1}$ Wakana Takahashi,,${ }^{1}$ and Takashi Ooi ${ }^{* 1}$ \\ ${ }^{1}$ Institute of Transformative Bio-Molecules (WPI-ITbM) and Department of Molecular and Macromolecular Chemistry, \\ Graduate School of Engineering, Nagoya University, Nagoya 464-8601, Japan.
}

\begin{abstract}
Catalysis by chiral weakly-coordinating anions (WCAs) remains underdeveloped due to the lack of a molecular design strategy for exploiting their characteristics, such as the non-nucleophilic nature. Here, we report the development of a chiral borate ion comprising an $O, N, N, O$-tetradentate backbone, which ensures hitherto unattainable structural robustness. Upon pairing with a proton, the hydrogen borate acts as an effective catalyst for the asymmetric Prins-type cyclization of vinyl ethers, providing access to structurally and stereochemically defined dihydropyrans. The key to selectivity control is the distinct ability of the borate ion to discriminate the prochiral faces of the acyclic oxonium ion intermediate and dictate the regiochemical outcome. We anticipate that this study paves the way for exploring the untapped potential of WCA catalysis for selective chemical synthesis.
\end{abstract}

\section{INTRODUCTION}

Prochiral cations occupy a uniquely important place in organic chemistry as reagents and reactive intermediates. In the bond formation of these cations with nucleophiles, precise discrimination of the enantiotopic faces is often required for obtaining stereochemically defined products. However, a reliable strategy for implementing such a general mode of absolute stereocontrol is underdeveloped. Ion pairing with a chiral anion is one of the simplest and most powerful approaches for imparting chirality to the cationic species, which is particularly true considering the advent of asymmetric chiral anion-directed catalysis (ACDC) and its rapid evolution over the last decade. ${ }^{1-3}$ The dominant strategy is based on the use of conjugate bases of Brønsted acids, wherein selection and structural modification of the chiral Brønsted acid itself or the organic scaffold that non-covalently binds to an achiral anion can endow the anions with a suitable chiral architecture and allow for tuning of their steric and electronic properties. The widespread utility of this approach has been demonstrated through the significant advancement in asymmetric organocatalysis, ${ }^{4}$ including anion phase-transfer ${ }^{5}$ and anion-binding catalysis ${ }^{6}$, and transition-metal catalysis, ${ }^{7,8}$ while manifesting the current limitations. An alternative yet distinct strategy for practicing ACDC is to exploit the intrinsic features of weakly-coordinating anions (WCAs).$^{9,10}$ WCA can form a stable ion pair with a cation without forming a covalent bond owing to its non-nucleophilic nature; hence, it has traditionally been employed as the counterion of highly electrophilic, labile cations. Although it is implied by the name that WCA shows weak interaction with the pairing cation, chiral WCAs can discriminate the chirality of the cation as they have been utilized as reagents for the separation of enantiomeric cationic compounds. ${ }^{11-14}$ These characteristics have encouraged the development of chiral WCAs that are competent for catalytically controlling reactive cationic intermediates, which in turn are attractive and advantageous for expanding the potential of ACDC. However, the pursuit of this approach 
has met with limited success. ${ }^{15-17}$

The borate ion such as tetraarylborate is a representative WCA that is widely used as the counterion for a variety of cationic species. In particular, tetraalkoxyborates $\left(\mathrm{B}(\mathrm{OR})_{4}^{-}\right)$constitute a venerable class of borates for developing chiral WCAs because these structurally diverse ions can be easily prepared by the condensation of boric acids (esters) with various chiral alcohols. Echoing with their ready accessibility, several chiral $\mathrm{B}(\mathrm{OR})_{4}^{-}$ions have been elaborated and evaluated as stereocontrollers following the pioneering contribution of Arndtsen, who used a binaphthol-derived chiral borate for asymmetric copper catalysis. ${ }^{18-24}$ Nevertheless, the rational development of boron-based chiral anions that can precisely control prochiral cations remains elusive. This is probably attributed to the inherent structural instability of $\mathrm{B}(\mathrm{OR})_{4}^{-}$resulting from the reversibility of boron-oxygen (B-O) bond formation, allowing the exchange of the oxy-ligand (OR), especially under acidic conditions. ${ }^{25-27}$ In fact, previously reported chiral borates, most of which adopt a bora-spiro core consisting of two bidentate chiral backbones, are not stable enough to be isolated by a standard purification procedure such as silica gel column chromatography; these borates are known to be structurally fluxional in solution in the absence of basic substrates such as imines or amines. ${ }^{28}$ These profiles, while crucial for the in situ assembly of thermodynamically favorable borate catalysts, reflect the lack of a strategy for the development of chiral borates with structural robustness and sufficient diversity for eliciting the untapped potential as chiral WCAs in the arena of asymmetric catalysis. Here, we describe a solution to this problem; that is, the molecular design, synthesis, and characterization of structurally robust chiral borates, which act as highly effective chiral WCA catalysts for achieving a highly regio- and enantioselective protonative Prins-type cyclization.

\section{RESULTS AND DISCUSSION}

The molecular design strategy was derived from our hypothesis on the origin of structural instability associated with the existing borate ions, which generally comprise bidentate chiral backbones (Fig. 1a). The most negatively polarized oxygen atom is susceptible to protonation, triggering the cleavage of the B-O bond with concomitant rotation around the $\mathrm{B}-\mathrm{X}$ axis to generate the corresponding trivalent borane with a vacant orbital. The facile nucleophilic attack of a Lewis base such as $\mathrm{H}_{2} \mathrm{O}$ to the vacant orbital leads to decomposition through hydrolysis or the formation of another borate derivative. With this consideration in mind, we reasoned that the use of a tetradentate backbone would provide a key structural element for rendering a borate ion stable, as it bridges two $\mathrm{X}$ atoms to restrict the rotation around the $\mathrm{B}-\mathrm{X}$ axis. Furthermore, the introduction of sterically demanding substituents to the appropriate positions could prevent the boron center from undergoing nucleophilic attack, thus delivering kinetic stabilization. The actual structure of borate $\mathbf{1}$ we designed is shown in Fig. 1b. This borate ion features an $O, N, N, O$-tetradentate backbone consisting of an axially chiral binaphthyl framework with a rigid amide linkage to the oxygen-attached carbon bearing geminal aromatic substituents, which allow not only steric protection but also modification of the chiral environment around the boron center. The synthesis of 1 was implemented in a straightforward manner, starting from the commercially available (R)-1,1'-binaphthyl-2,2'-diamine (Fig. 1b). After initial acylation of the nitrogen atom with ethyl chlorooxoacetate, the resulting bisamide $\mathbf{2}$ was exposed to an aryl Grignard reagent to install the aromatic appendages, affording $C_{2}$-symmetric amide alcohol 3 in good yield. Subsequent treatment of $\mathbf{3}$ with trimethyl borate in acetonitrile at $90{ }^{\circ} \mathrm{C}$ in the presence of triethylamine furnished the 
requisite borate salt $\mathbf{1} \cdot \mathrm{HNEt}_{3}$. As expected, triethylammonium borate $\mathbf{1} \cdot \mathrm{HNEt}_{3}$ had sufficient structural robustness to be isolated by standard silica gel column chromatography. The three-dimensional molecular architecture of 1a $(\mathrm{Ar}=\mathrm{Ph})$ was unambiguously determined by single-crystal X-ray diffraction analysis of its benzyldimethylammonium salt, uncovering the unique boron-sharing 5:7:5-fused tricyclic core; the two planes constructed by the NBO connection were nearly perpendicular to each other (Fig. 1c and Fig. S2). From this core structure, four phenyl groups spread outward to create a discrete chiral environment over the boron center. Importantly, one of the carbonyl oxygens of the amide moieties interacted with the pairing benzyldimethylammonium cation via a weak hydrogen bond $\left(\mathrm{C}=\mathrm{O} \cdots \mathrm{HN}^{+}=1.727 \AA\right)$ so that the cation is positioned between a phenyl group and a naphthyl group (Fig. 1c-iii).

(a)

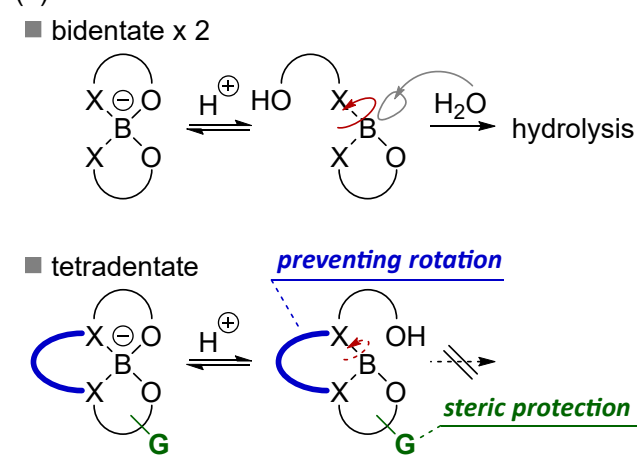

(b)<smiles>CCOC(=O)C(=O)Nc1ccc2ccccc2c1-c1c(NC(=O)C(=O)OCC)ccc2ccccc12</smiles>

$0{ }^{\circ} \mathrm{C} \sim \mathrm{rt}$

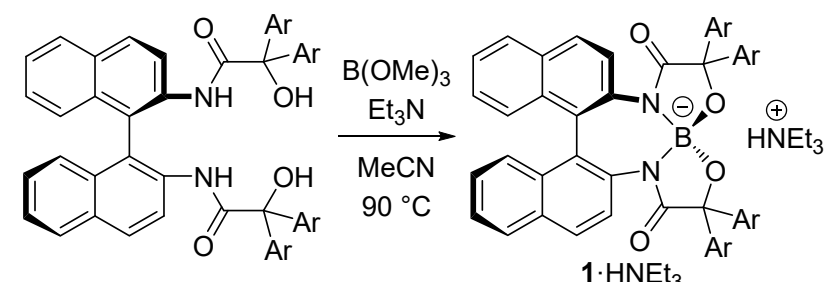

(c)

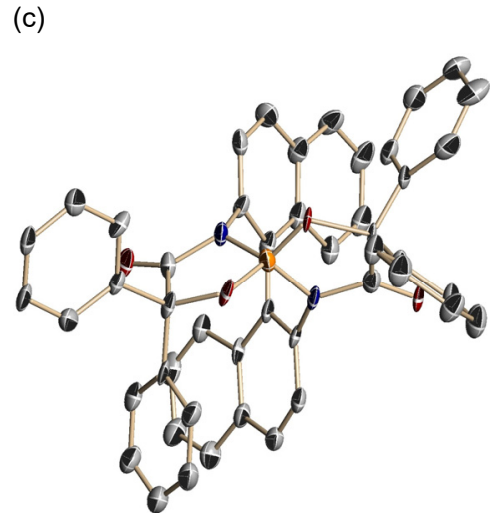

i) front view of 1a

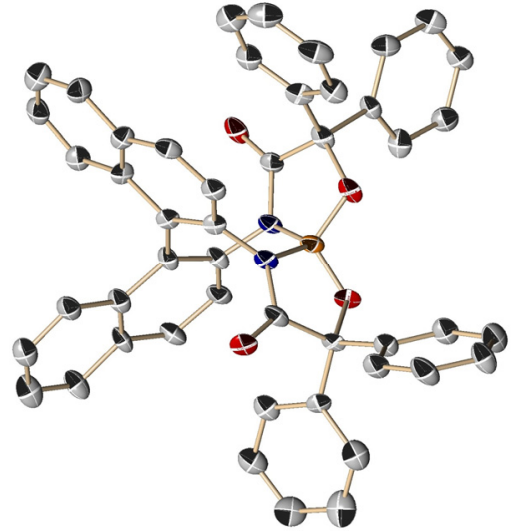

ii) side view of $1 \mathrm{a}$

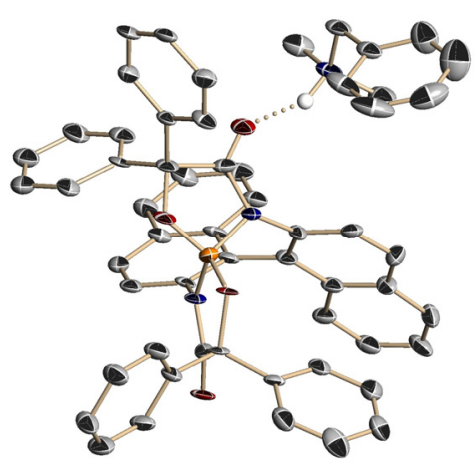

iii) $1 \mathrm{a} \cdot \mathrm{HNMe}_{2} \mathrm{Bn}$

Fig. 1 (a) Hydrolysis of borates and design concept for structurally robust borates, (b) Molecular structure and synthesis of chiral borate $\mathbf{1} \cdot \mathrm{HNEt}_{3}$. (c) ORTEP diagrams of chiral benzyldimethylammonium borate $\mathbf{1 a} \cdot \mathrm{HNMe}_{2} \mathrm{Bn}(50 \%$ probability, Calculated hydrogen atoms are omitted for clarity.).

The structural robustness of $\mathbf{1}$ prompted us to prepare the corresponding hydrogen borate $\mathbf{1} \cdot \mathrm{H}$ by an ordinary ion-exchange method for use as a chiral Brønsted acid. Thus, an ethanolic solution of $\mathbf{1} \cdot \mathrm{HNEt}_{3}$ was allowed to pass through a cation-exchange resin column (Amberlite IR-120 $\left(\mathrm{H}^{+}\right.$form)), and the eluent was concentrated to afford $\mathbf{1} \cdot \mathrm{H}$ in essentially pure form. Although the $\mathrm{p} K_{\mathrm{a}}$ value of the ionic acid could not be directly compared with that of non-ionic Brønsted acids, the calculated $\mathrm{p} K_{\mathrm{a}}$ of $1 \mathbf{a} \cdot \mathrm{H}\left(9.9\right.$ in $\mathrm{MeCN}$, cf.: 8.7 for $\mathrm{H}_{2} \mathrm{SO}_{4}$ ) revealed its high acidity. ${ }^{29,30}$ Furthermore, the self-consistent hydrogen-bond acceptor parameter $(\beta)$, introduced by 
Hunter, was estimated to be 7.6, which is comparable to that of bis(trifluoromethanesulfonyl)imidate $\left(\beta=7.3, \mathrm{p} K_{\mathrm{a}}=\right.$ 0.3 for $\left.\left(\mathrm{CF}_{3} \mathrm{SO}_{3}\right)_{2} \mathrm{NH}\right),{ }^{31,32}$ indicating the weakly-coordinating character of $\mathbf{1} \mathbf{a}$ in pairing with a cation such as a proton.

As a reaction platform suitable for evaluating the catalytic performance of $1 \cdot \mathrm{H}$, we set a Prins-type cyclization of vinylic ethers $2 .^{33-38}$ This transformation involving the protonative generation of an oxonium ion, followed by intramolecular carbon-carbon bond formation and final deprotonation from the transient carbocation, is of significant synthetic relevance as it offers straightforward access to substituted dihydropyrans $\mathbf{3} \mathbf{4}$, which are valuable six-membered oxygen heterocycles (Fig. 2a, Scheme). However, the available catalytic protocols are scarce, ${ }^{39}$ and precise control of the regio- and stereochemical outcome remains a formidable challenge for realizing the full potential of this reaction system. Our incipient expectation was that the chiral hydrogen borate $\mathbf{1} \cdot \mathrm{H}$ would be sufficiently acidic to generate the intermediary oxonium ion and that its prochiral faces could be discriminated by the pairing 1 in the cyclization event. As the initial attempt, vinyl ether $2 \mathbf{a}$ was treated with $5 \mathrm{~mol} \%$ of $1 \mathrm{a} \cdot \mathrm{H}$ in chloroform $\left(\mathrm{CHCl}_{3}\right)$ at $-60{ }^{\circ} \mathrm{C}$, which gave a mixture of regioisomeric dihydropyrans $3 \mathbf{a}$ and $\mathbf{4 a}(\mathbf{3 a} / \mathbf{4 a}=6: 1)$ in $35 \%$ yield (Fig. 2a, entry 1). The enantiomeric excess (ee) of the major product $3 \mathbf{a}$ was determined to be $55 \%$ by chiral-

(a)

(5)

(b)
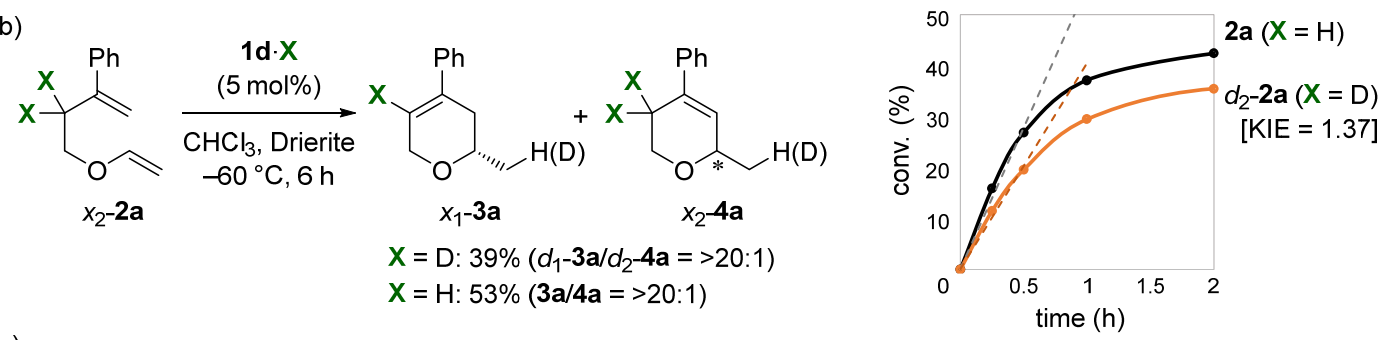

(c)

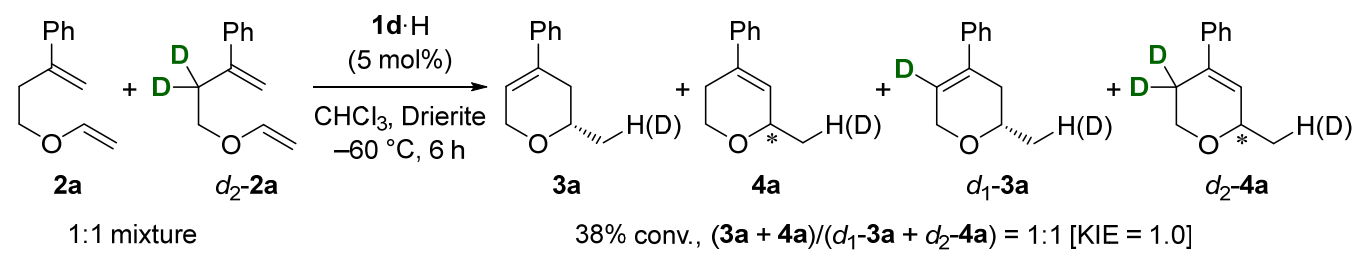

Fig. 2 (a) Catalyst optimization. The reaction was performed in $0.1 \mathrm{mmol}$ scale with $\mathbf{2 a}$ and $5 \mathrm{~mol} \%$ of $\mathbf{1} \cdot \mathrm{H}^{\text {in }} \mathrm{CHCl}_{3}(0.1$ M) at $-60{ }^{\circ} \mathrm{C}$ for $24 \mathrm{~h}$. Isolated yield was indicated. The regioisomeric ratio (rr) was assigned by ${ }^{1} \mathrm{H} \mathrm{NMR}(500 \mathrm{MHz})$ analysis of the crude aliquot. The enantiomeric excess (ee) of the major isomer was determined by HPLC with DAICEL CHIRALCEL OJ3 as a chiral stationary phase and hexane/EtOH $=99: 1$ as eluent. Drierite $=$ porous anhydrous $\mathrm{CaSO}_{4} .(\mathrm{b})$ and (c) Determination of the kinetic isotope effect (KIE) at the deprotonation stage. Conversions and isomeric ratios were determined by ${ }^{1} \mathrm{H}$ NMR (500 MHz) analysis of the crude aliquots containing trimethylsilylbenzene as an internal standard (average of three runs). 
stationary-phase HPLC analysis. This promising result encouraged us to examine the effect of the catalyst structure on reactivity and selectivity, specifically, with respect to the steric and electronic properties of the aromatic substituents. While replacement of the phenyl groups with 4-tolyl groups (1b) subtly affected the reaction profile, the use of 1c bearing 3,5-xylyl groups led to a marked improvement in both stereoselectivity and catalytic efficiency (entries 2 and 3). An additional intriguing and important observation was that the regioselectivity was also significantly improved in response to the catalyst modification, indicating that the borate ion is responsible for not only the stereo-determining step but also the product-determining deprotonation step (vide infra). Eventually, the use of 1d with 3,5-di- $n$-butylphenyl groups resulted in the exclusive production of 3a in $77 \%$ yield with $94 \%$ ee (entry 4). The remaining problem was the insufficient chemical yield, which was likely due to the formation of hydration-related side products and was solved by performing the reaction in the presence of the desiccant Drierite (porous anhydrous calcium sulfate) under otherwise identical conditions; 3a was obtained in 95\% yield without any detrimental effect on the selectivity (entry 5). Moreover, 1 was easily recovered as a triethylammonium salt (1d $\cdot \mathrm{HNEt}_{3}$ ) during product purification after quenching the reaction by the addition of triethylamine $\left(\mathrm{NEt}_{3}\right)$.

To gain insight into the origin of the catalyst-dependent remarkable enhancement of regioselectivity in the final deprotonation from the transient carbocation, we performed the reaction with deuterated vinyl ether $d_{2}-\mathbf{2 a}$ (Fig. $2 b)$. At the outset, we sought to confirm the intrinsic regiochemical preference by the use of $( \pm)$-10-camphorsulfonic acid as a simple catalyst ( 5 mol\%), which gave a mixture of $d_{1}-\mathbf{3 a}$ and $d_{2}-\mathbf{4 a}$ in a ratio of 0.8:1 (Fig. S1) probably because of the preferable deprotonation over dedeuteration as the opposite preference was observed in the reaction of $\mathbf{2 a}$ under otherwise identical conditions $(\mathbf{3} \mathbf{a} / \mathbf{4} \mathbf{a}=1.7: 1)$. In sharp contrast, exposing $d_{2}-\mathbf{2} \mathbf{a}$ to the standard conditions with deuterated catalyst $\mathbf{1 d} \cdot \mathrm{D}$ for $6 \mathrm{~h}$ afforded $d_{1}-\mathbf{3 a}$ exclusively $\left(d_{1}-\mathbf{3 a} / d_{2}-\mathbf{4 a}=>20: 1\right)$, identical regioselectivity observed in the separate reaction of $\mathbf{2 a}$ with $\mathbf{1 d} \cdot \mathrm{H}$ as a catalyst under similar conditions (Fig. 2b). The selective formation of $d_{1}-\mathbf{3 a}$ through dedeuteration in complete preference over deprotonation to give $d_{2}-\mathbf{4 a}$ signified that the deprotonation (dedeuteration) step proceeded in a catalyst-controlled manner rather than by spontaneous release. Interestingly, the conversion of $d_{2}-\mathbf{2 a}$ was lower than that of $\mathbf{2 a}$, as revealed by ${ }^{1} \mathrm{H}$ NMR analysis $(500 \mathrm{MHz})$ of the crude mixture containing trimethylsilylbenzene as the internal standard. This observation led us to evaluate the primary kinetic isotope effect (KIE) in these parallel reactions, and the KIE for $\mathbf{2 a} / d_{2}-\mathbf{2 a}$ was determined to be 1.37.40,41 Judging from the deuterium incorporation into the methyl group of the products (ca. $40 \%$ by $500 \mathrm{MHz}{ }^{1} \mathrm{H}$ NMR analysis) and the magnitude of the KIE, the rate difference presumably stems from the lower acidity of the deuterated catalyst $\mathbf{1 d} \cdot \mathrm{D},{ }^{42,43}$ implying that the actual KIE would be negligible. We also evaluated the KIE in the intermolecular competition reaction with an equimolar mixture of 2a and $d_{2}$-2a under otherwise similar conditions (Fig. 2c). While there was a slight decrease in the overall reaction rate, the conversions of $2 \mathbf{a}$ and $d_{2}$-2a were nearly equal and almost equimolar amounts of $\mathbf{3 a} / \mathbf{4} \mathbf{a}$ and $d_{1}-\mathbf{3 a} / d_{2}-\mathbf{4 a}$ were obtained (extent of deuterium incorporation into the methyl groups was $<10 \%$ ), thereby providing a KIE close to 1.0. These results suggested that the deprotonation does not participate in the rate-limiting process and that carbon-carbon bond formation is most likely the rate-determining step. In addition, the constantly observed excellent regioselectivity emphasized that the catalyst-controlled deprotonation plays a critical role in dictating the reaction pathway to give the dihydropyran product 3 .

Having identified the optimal borate structure and reaction conditions, we investigated the substrate 
generality of the 1d·H-catalyzed Prins-type cyclization of vinylic ethers 2 (Table 1). As shown in Table 1, the reaction was tolerant to the incorporation of aromatic substituents (Ar') with different electronic attributes, and the corresponding dihydropyrans $\mathbf{3}$ were obtained predominantly with a high level of enantiocontrol, while slightly lower regio- and stereoselectivities were observed in the cyclizations with $\mathbf{2}$ having meta-substituted phenyl groups (entries $1 \sim 5)$. In the reactions with substituted vinyl ethers, only $Z$-configured isomers underwent intramolecular bond formation to give 3 in good yield with high stereoselectivity, probably because $E$-isomers were resistant to the initial protonation by $\mathbf{1 d} \cdot \mathrm{H}$ (entries 6 9). Vinylic ethers with a substituent at the terminal carbon of the nucleophilic olefinic component were amenable to this protocol, and 2,3-disubstituted dihydropyrans $\mathbf{3} \mathbf{k} \sim \mathbf{n}$ were obtained with virtually complete diastereoselectivity and excellent enantioselectivity (entries 10 13). Notably, the olefin geometry was again important, and the $E$-configuration was crucial for smooth ring closure.

Table 1 Substrate generality. ${ }^{\mathrm{a}}$

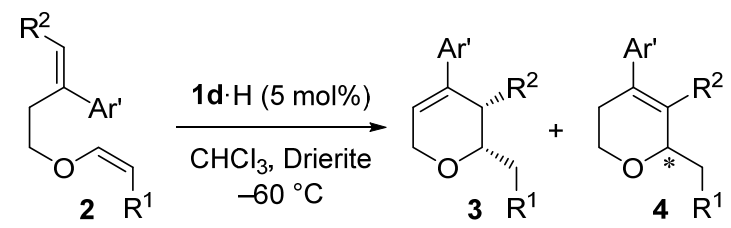

\begin{tabular}{lllllllllll} 
entry & $\mathrm{Ar}$ & $\mathrm{R}^{1}$ & $\mathrm{R}^{2}$ & $\mathbf{2}$ & time $(\mathrm{h})$ & yield $(\%)^{b}$ & $\mathrm{rr}^{c}$ & $\mathrm{dr}^{c}$ & ee (\%) & Prod \\
\hline 1 & $4-\mathrm{MeC}_{6} \mathrm{H}_{4}$ & $\mathrm{H}$ & $\mathrm{H}$ & $\mathbf{2 b}$ & 24 & 97 & $19: 1$ & - & 93 & $\mathbf{3 b}$ \\
2 & $4-\mathrm{ClC}_{6} \mathrm{H}_{4}$ & $\mathrm{H}$ & $\mathrm{H}$ & $\mathbf{2 c}$ & 24 & 82 & $>20: 1$ & - & 95 & $\mathbf{3 c}$ \\
3 & $4-\mathrm{FC}_{6} \mathrm{H}_{4}$ & $\mathrm{H}$ & $\mathrm{H}$ & $\mathbf{2 d}$ & 24 & 98 & $>20: 1$ & - & 94 & $\mathbf{3 d}$ \\
4 & $3-\mathrm{MeC}_{6} \mathrm{H}_{4}$ & $\mathrm{H}$ & $\mathrm{H}$ & $\mathbf{2 e}$ & 72 & 84 & $10: 1$ & - & 90 & $\mathbf{3 e}$ \\
5 & $3-\mathrm{MeOC}_{6} \mathrm{H}_{4}$ & $\mathrm{H}$ & $\mathrm{H}$ & $\mathbf{2 f}$ & 48 & 73 & $17: 1$ & - & 88 & $\mathbf{3 f}$ \\
6 & $\mathrm{Ph}$ & $\mathrm{Me}$ & $\mathrm{H}$ & $\mathbf{2 g}$ & 24 & 87 & $>20: 1$ & - & 93 & $\mathbf{3 g}$ \\
7 & $4-\mathrm{FC}_{6} \mathrm{H}_{4}$ & $\mathrm{Me}$ & $\mathrm{H}$ & $\mathbf{2 h}$ & 72 & 98 & $>20: 1$ & - & 90 & $\mathbf{3 h}$ \\
8 & $4-\mathrm{MeC}_{6} \mathrm{H}_{4}$ & $\mathrm{Me}$ & $\mathrm{H}$ & $\mathbf{2 i}$ & 24 & 89 & $>20: 1$ & - & 89 & $\mathbf{3 i}$ \\
9 & $\mathrm{Ph}_{10}$ & $\mathrm{Et}$ & $\mathrm{H}$ & $\mathbf{2 j}$ & 48 & 79 & $>20: 1$ & - & 94 & $\mathbf{3 j}$ \\
10 & $\mathrm{Ph}_{11}^{4}$ & $\mathrm{H}$ & $\mathrm{Me}$ & $\mathbf{2 k}$ & 24 & 92 & $>20: 1$ & $>20: 1$ & 94 & $\mathbf{3 k}$ \\
12 & $3-\mathrm{ClC}_{6} \mathrm{H}_{4}$ & $\mathrm{H}$ & $\mathrm{Me}$ & $\mathbf{2 l}$ & 24 & 74 & $>20: 1$ & $>20: 1$ & 93 & $\mathbf{3 1}$ \\
13 & $\mathrm{Ph}^{-\mathrm{MeC}_{6} \mathrm{H}_{4}}$ & $\mathrm{H}$ & $\mathrm{Me}$ & $\mathbf{2 m}$ & 24 & 96 & $>20: 1$ & $>20: 1$ & 90 & $\mathbf{3 m}$ \\
\hline
\end{tabular}

${ }^{a}$ The reaction was performed in $0.1 \mathrm{mmol}$ scale with 2 and $5 \mathrm{~mol} \%$ of $\mathbf{1 d} \cdot \mathrm{H}$ in $\mathrm{CHCl}_{3}(0.1 \mathrm{M})$ at $-60{ }^{\circ} \mathrm{C} .{ }^{b}$ Isolated yield was indicated. ${ }^{c}$ The regioisomeric ratio (rr) and diastereomeric ratio (dr) were assigned by ${ }^{1} \mathrm{H}$ NMR (500 MHz) analysis of the crude aliquot. ${ }^{d}$ The enantiomeric excess (ee) of the major isomer was determined by HPLC equipped with a chiral column.

The stereochemically defined dihydropyran products $\mathbf{3}$ serve as versatile synthetic intermediates, as they can be derivatized into various oxygen-containing six-membered heterocycles in a straightforward manner with preservation of the stereochemical integrity, thereby demonstrating the utility of the catalytic asymmetric Prins-type cyclization protocol (Fig. 3). The olefin component of $\mathbf{3}$ could be harnessed as a functional handle to increase stereochemical and structural complexity. For example, simple hydrogenation of $\mathbf{3 k}$ with palladium on charcoal 
$(\mathrm{Pd} / \mathrm{C})$ afforded tetrahydropyran $\mathbf{5}$ possessing three consecutive stereogenic centers in good yield with reasonably high diastereoselectivity, and the hydroboration-oxidation sequence allowed the introduction of a hydroxy group with a similar degree of diastereocontrol. Alcohol $\mathbf{6}$ was subjected to single-crystal X-ray diffraction analysis after conversion to ferrocenyl carboxylate, and the absolute configuration of $\mathbf{3} \mathbf{k}$ was established as $2 S, 3 S$ at this stage (Fig. S3). Moreover, single-electron oxidation of 3k under the photoredox conditions facilitated carbon-carbon bond formation at the 5-position of the tetrahydropyran, although the corresponding ketone 7 was obtained as a mixture of diastereomers. ${ }^{44}$ On the other hand, treatment of $\mathbf{3 k}$ with pyridinium dichromate (PDC) promoted allylic oxidation to give unsaturated lactone $\mathbf{8}$ in good yield. ${ }^{45}$ The allylic methylene protons are sufficiently acidic to be deprotonated by the $n$-butyl lithium/tetramethylethylenediamine (TMEDA) system to generate a carbanion that can be trapped by an electrophile such as a Weinreb amide to provide acylated dihydropyran 9 in stereochemically pure form. ${ }^{46}$ Hydride abstraction at the allylic position was also feasible by the action of the in situ generated trityl cation, and the intermediary oxonium ion reacted with an allylsilane to furnish tetrasubstituted dihydropyran $\mathbf{1 0}$ with complete diastereoselectivity. ${ }^{47}$

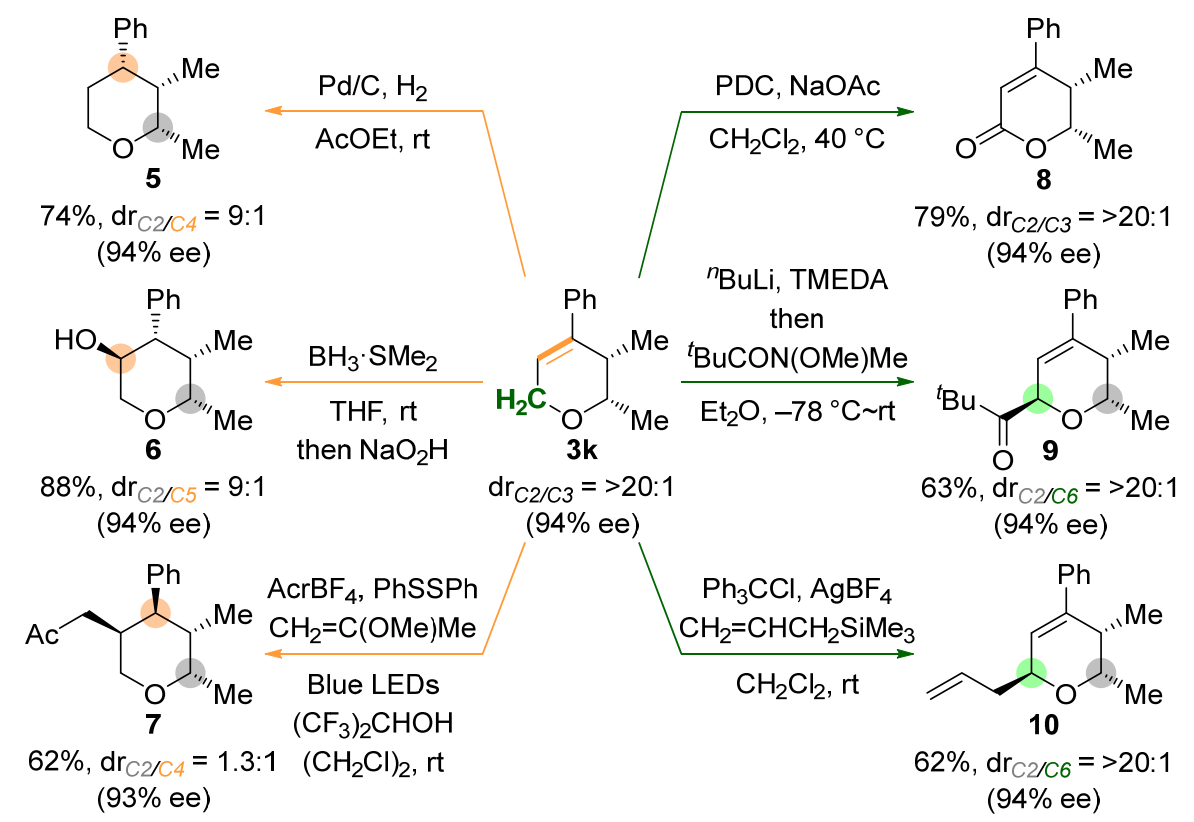

Figure 3 Derivatization of dihydropyran 3k. Acr $=N$-phenyl-9-mesitylacridinium

\section{CONCLUSIONS}

We have introduced a molecular design strategy for the rational development of a structurally robust chiral borate ion based on the use of an $O, N, N, O$-tetradentate backbone. X-ray crystallographic and theoretical analyses reveal the unique structural features and weakly-coordinating character of this borate ion. Furthermore, we have clearly demonstrated that the corresponding chiral hydrogen borate acts as an effective catalyst for the highly regio- and stereoselective Prins-type cyclization of vinyl ethers, where the borate ion precisely discriminates the prochiral oxonium ion intermediate and rigorously dictates the final deprotonation pathway. The structurally and stereochemically defined dihydropyran products can serve as versatile synthetic building blocks. We believe that the present study shows a new direction for the development of chiral WCAs capable of catalytically controlling 
prochiral cations for achieving otherwise difficult stereoselective chemical transformations.

\section{ACKNOWLEDGEMENT}

Financial support was provided by CREST-JST (JPMJCR13L2: 13418441, to TO), a Grant-in-Aid for Scientific Research on Innovative Areas "Hybrid Catalysis" (No. 17H06444, to TO), and Grants of JSPS for Scientific Research. N.T. and T.K. acknowledges JSPS for financial support. Program for Leading Graduate Schools "Integrative Graduate Education and Research Program in Green Natural Sciences" in Nagoya University and "Graduate Program of Transformative Chem-Bio Research" in Nagoya University, supported by MEXT (WISE Program) are acknowledged.

\section{REFERENCES}

(1) R. J. Phipps, G. L. Hamilton, F. D. Toste, The Progression of Chiral Anions from Concepts to Applications in Asymmetric Catalysis, Nat. Chem. 2012, 4, 603-614.

(2) M. Mahlau, B. List, Asymmetric Counteranion-Directed Catalysis: Concept, Definition, and Applications, Angew. Chem. Int. Ed. 2013, 52, 518-533.

(3) K. Brak, E. N. Jacobsen, Asymmetric Ion-Pairing Catalysis, Angew. Chem. Int. Ed. 2013, 52, 534-561.

(4) S. Mayer, B. List, Asymmetric Counteranion-Directed Catalysis, Angew. Chem. Int. Ed. 2006, 45, 4193-4195.

(5) V. Rauniyar, A. D. Lackner, G. L. Hamilton, F. D. Toste, Asymmetric electrophilic fluorination using an anionic chiral phase-transfer catalyst, Science 2011, 334, 1681-1684.

(6) I. T. Raheem, P. S. Thiara, E. A. Peterson, E. N. Jacobsen, Enantioselective Pictet-Spengler-Type Cyclizations of Hydroxylactams: H-Bond Donor Catalysis by Anion Binding, J. Am. Chem. Soc. 2007, 129, 13404-13405.

(7) V. Komanduri, M. J. Krische, Enantioselective Reductive Coupling of 1,3-Enynes to Heterocyclic Aromatic Aldehydes and Ketones via Rhodium Catalyzed Asymmetric Hydrogenation: Mechanistic Insight into the Role of Brønsted Acid Additives, J. Am. Chem. Soc. 2006, 128, 16448-16449.

(8) G. L. Hamilton, E. J. Kang, M. Mba, F. D. Toste, A Powerful Chiral Counterion Strategy for Asymmetric Transition Metal Catalysis, Science 2007, 317, 496-499.

(9) I. Krossing, I. Raabe, Noncoordinating Anions-Fact or Fiction? A Survey of Likely Candidates, Angew. Chem. Int. Ed. 2004, 43, 2066-2090.

(10) I. M. Riddlestone, A. Kraft, J. Schaefer, I. Krossing, Taming the Cationic Beast: Novel Developments in the Synthesis and Application of Weakly Coordinating Anions, Angew. Chem. Int. Ed. 2018, 57, 13982-14024.

(11) F. Freire, E. Quiñoá, R. Riguera, In tube Determination of the Absolute Configuration of $\alpha$ - and $\beta$-Hydroxy Acids by NMR via Chiral BINOL Borates, Chem. Commun. 2008, 4147-4149.

(12) M. Akabane, A. Yamamoto, S.-i. Aizawa, A. Taga, S. Kodama, Simultaneous Enantioseparation of Monosaccharides Derivatized with L-Tryptophan by Reversed Phase HPLC, Anal. Sci. 2014, 30, 739-743.

(13) L. W.-Y. Wong, G. S.-S. Tam, X. Chen, F. T.-K. So, A. Soecipto, F. K. Sheong, H. H.-Y. Sung, Z. Lin, I. D. Williams, A Chiral Spiroborate Anion from Diphenyl-L-Tartramide $\left[\mathrm{B}\left\{\mathrm{L}-\operatorname{Tar}(\mathrm{NHPh})_{2}\right\}_{2}\right]^{-}$Applied to Some Challenging Resolutions, CrystEngComm 2018, 20, 4831-4848.

(14) E. Tayama, T. Sugawara, Chiral Tetraaryl- and Tetraalkynylborates as Chiral Solvating Agents for 
Tetraalkylammonium Salts, Eur. J. Org. Chem. 2019, 803-811.

(15) P. Pommerening, J. Mohr, J. Friebel, M. Oestreich, Synthesis of a Chiral Borate Counteranion, Its Trityl Salt, and Application Thereof in Lewis-Acid Catalysis, Eur. J. Org. Chem. 2017, 2312-2316.

(16) D. Uraguchi, H. Sasaki, Y. Kimura, T. Ito, T. Ooi, Molecular Design, Synthesis, and Asymmetric Catalysis of a Hexacoordinated Chiral Phosphate Ion, J. Am. Chem. Soc. 2018, 140, 2765-2768.

(17) H.-J. Jiang, X.-M. Zhong, J. Yu, Y. Zhang, X. Zhang, Y.-D. Wu, L.-Z. Gong, Assembling a Hybrid Pd Catalyst from a Chiral Anionic $\mathrm{Co}^{\mathrm{III}}$ Complex and Ligand for Asymmetric $\mathrm{C}\left(\mathrm{sp}^{3}\right)$-Functionalization, Angew. Chem. Int. Ed. 2019, 58, 1803-1807.

(18) D. B. Llewellyn, D. Adamson, B. A. Arndtsen, A Novel Example of Chiral Counteranion Induced Enantioselective Metal Catalysis: The Importance of Ion-Pairing in Copper-Catalyzed Olefin Aziridination and Cyclopropanation, Org. Lett. 2000, 2, 4165-4168.

(19) G. Hu, L. Huang, R. H. Huang, W. D. Wulff, Evidence for a Boroxinate Based Brønsted Acid Derivative of VAPOL as the Active Catalyst in the Catalytic Asymmetric Aziridination Reaction, J. Am. Chem. Soc. 2009, 131, 15615-15617.

(20) G. Hu, A. K. Gupta, R. H. Huang, M. Mukherjee, W. D. Wulff, Substrate-Induced Covalent Assembly of a Chemzyme and Crystallographic Characterization of a Chemzyme-substrate Complex, J. Am. Chem. Soc. 2010, 132, 14669-14675.

(21) H. Ren, W. D. Wulff, Direct Catalytic Asymmetric Aminoallylation of Aldehydes: Synergism of Chiral and Non Chiral Brønsted Acids, J. Am. Chem. Soc. 2011, 133, 5656-5659.

(22) T. Hashimoto, A. O. Gálvez, K. Maruoka, In Situ Assembled Boronate Ester Assisted Chiral Carboxylic Acid Catalyzed Asymmetric Trans-Aziridinations, J. Am. Chem. Soc. 2013, 135, 17667-17670.

(23) W. Zhao, L. Huang, Y. Guan, W. D. Wulff, Three-Component Asymmetric Catalytic Ugi Reaction-Concinnity from Diversity by Substrate-Mediated Catalyst Assembly, Angew. Chem. Int. Ed. 2014, 53, 3436-3441.

(24) M. Ueda, Y. Yagyu, I. Ryu, Ammonium Chiral Borate Salt Catalyzed Asymmetric Friedel-Crafts Alkylation of Indoles with $\alpha, \beta$-Disubstituted Enals, Tetrahedron: Asymmetry 2017, 28, 1070-1077.

(25) K. Ishihara, H. Yamamoto, Brønsted Acid Assisted Chiral Lewis Acid (BLA) Catalyst for Asymmetric DielsAlder Reaction, J. Am. Chem. Soc. 1994, 116, 1561-1562.

(26) K. Ishihara, M. Miyata, K. Hattori, T. Tada, H. Yamamoto, A New Chiral BLA Promoter for Asymmetric Aza Diels-Alder and Aldol-Type Reactions of Imines, J. Am. Chem. Soc. 1994, 116, 10520-10524.

(27) A. K. Gupta, X. Yin, M. Mukherjee, A. A. Desai, A. Mohammadlou, K. Jurewicz, W. D. Wulff, Catalytic Asymmetric Epoxidation of Aldehydes with Two VANOL-Derived Chiral Borate Catalysts, Angew. Chem. Int. Ed. 2019, 58, 3361-3367.

(28) G. Hu, A. K. Gupta, L. Huang, W. Zhao, X. Yin, W. E. G. Osminski, R. H. Huang, W. D. Wulff, J. A. Izzo,; M. J. Vetticatt, Pyro-Borates, Spiro-Borates, and Boroxinates of BINOL-Assembly, Structures, and Reactivity, J. Am. Chem. Soc. 2017, 139, 10267-10285.

(29) A. Kütt, T. Rodima, J. Saame, E. Raamat, V. Mäemets, I. Kaljurand, I. A. Koppel, R. Y. Garlyauskayte, Y. L. Yagupolskii, L. M. Yagupolskii, E. Bernhardt, H. Willner, I. Leito, Equilibrium Acidities of Superacids, J. Org. Chem. 2011, 76, 391-395. 
(30) K. Ohmatsu, T. Nakashima, M. Sato, T. Ooi, Direct Allylic C-H Alkylation of Enol Silyl Ethers Enabled by Photoredox-Brønsted Base Hybrid Catalysis, Nat. Commun. 2019, 10, 2706.

(31) S. J. Pike, J. J. Hutchinson, C. A. Hunter, H-Bond Acceptor Parameters for Anions, J. Am. Chem. Soc. 2017, 139, 6700-6706.

(32) Z. Lu, J. Han, O. E. Okoromoba, N. Shimizu, H. Amii, C. F. Tormena, G. B. Hammond, B. Xu, Predicting Counterion Effects Using a Gold Affinity Index and a Hydrogen Bonding Basicity Index, Org. Lett. 2017, 19, 5848-5851.

(33) C. Olier, M. Kaafarani, S. Gastaldi, M. P. Bertrand, Synthesis of Tetrahydropyrans and Related Heterocycles via Prins Cyclization; Extension to Aza-Prins Cyclization, Tetrahedron 2010, 66, 413-445.

(34) G. C. Tsui, L. Liu, B. List, The Organocatalytic Asymmetric Prins Cyclization, Angew. Chem. Int. Ed. 2015, 54, 7703-7706.

(35) L. Liu, P. S. J. Kaib, A. Tap, B. List, A General Catalytic Asymmetric Prins Cyclization, J. Am. Chem. Soc. 2016, $138,10822-10825$.

(36) J. Liu, L. Zhou, C. Wang, D. Liang, Z. Li, Y. Zou, Q. Wang, A. Goeke, Catalytic Asymmetric Prins Bicyclization for the endo-Selective Formation of 2,6-dioxabicyclo[2.2.2]octanes, Chem. Eur. J. 2016, 22, 6258-6261.

(37) B. V. S. Reddy, P. N. Nair, A. Antony, N. Srivastava, Recent Advances in Prins Spirocyclization, Eur. J. Org. Chem. 2017, 5484-5496.

(38) L. Lempenauer, G. Lemière, E. Duñach, Cyclisation Reactions involving Alkyl Enol Ethers, Adv. Synth. Catal. 2019, 361, 5284-5304.

(39) A. Puglisi, A.-L. Lee, R. R. Schrock, A. H. Hoveyda, Operationally Simple, Efficient, and Diastereoselective Synthesis of cis-2,6-Disubstituted-4-Methylene Tetrahydropyrans Catalyzed by Triflic Acid, Org. Lett. 2006, 8, 1871-1874.

(40) E. M. Simmons, J. F. Hartwig, On the Interpretation of Deuterium Kinetic Isotope Effects in C-H Bond Functionalizations by Transition-Metal Complexes, Angew. Chem. Int. Ed. 2012, 51, 3066-3072.

(41) R. S. Klausen, C. R. Kennedy, A. M. Hyde, E. N. Jacobsen, Chiral Thioureas Promote Enantioselective PictetSpengler Cyclization by Stabilizing Every Intermediate and Transition State in the Carboxylic Acid-Catalyzed Reaction, J. Am. Chem. Soc. 2017, 139, 12299-12309.

(42) E. L. Wehry, L. B. Rogers, Deuterium Isotope Effects on the Protolytic Dissociation of Organic Acids in Electronically Excited States, J. Am. Chem. Soc. 1966, 88, 351-354.

(43) N. Mora-Diez, Y. Egorova, H. Plommera, P. R. Tremaine, Theoretical Study of Deuterium Isotope Effects on Acid-base Equilibria under Ambient and Hydrothermal Conditions, RSC Adv. 2015, 5, 9097-9109.

(44) F. Wu, L. Wang, J. Chen, D. A. Nicewicz, Y. Huang, Direct Synthesis of Polysubstituted Aldehydes via VisibleLight Catalysis, Angew. Chem. Int. Ed. 2018, 57, 2174-2178.

(45) G. V. M. Sharma, S. Mallesham, Stereoselective Total Synthesis of Styryl-Lactones: (+)-Crassalactones B and $\mathrm{C},(+)$-Howiionol A, (+)-Tricinnamate, $(+)$-Goniofufurone and (+)-Dicinnamoyl Goniofufurone, Tetrahedron: Asymmetry 2010, 21, 2646-2658.

(46) V. Rautenstrauch, Sigmatropic Reactions in Carbanions. I. The 5,6-Dihydro-2H-pyran-2-ide Cyclopropylenolate Rearrangement, Helv. Chim. Acta 1972, 55, 594-609. 
(47) Z. Wan, M. Meng, H. Lou, L. Liu, Practical and Highly Selective C-H Functionalization of Structurally Diverse Ethers, Angew. Chem. Int. Ed. 2014, 53, 13845-13849. 